\title{
Visual vertigo treatment through optokinetic stimulation with stationary anchoring
}

\author{
Chih-Pei Chang ${ }^{*}$ and Timothy C Hain ${ }^{2}$ \\ ${ }^{1}$ Department of Atmospheric Sciences, National Taiwan University, Taipei, Taiwan and Graduate School of Engineering and Applied Sciences, Naval Postgraduate \\ School Code MR/Cp, Monterey, CA 93943-5114, USA \\ ${ }^{2}$ Departments of Neurology, Otolaryngology, and Physical Therapy and Human Movement Science, Northwestern University, Chicago, Illinois 60611-3008, USA
}

\begin{abstract}
A theoretical framework to incorporate a stationary anchor in the repeated optokinetic stimulation to treat dizziness patients with visual vertigo is considered. The approach is opposite to the traditional treatment of avoiding triggers of dizziness by gaze stabilization. It emphasizes a gradually expanding but limited field of view in contrast to the full immersion or wide field of view typical of virtual reality treatment schemes. The advantages of this approach are elucidated by comparing with the mechanism of an adaptive filter (Kalman filter), and its application in assimilating different sources of imperfect meteorological observation data in numerical weather prediction.

In the stationary anchor approach the foreground contains a higher velocity that provides strong optical simulation and the resulting estimate may provoke a dizziness attack, while the background with weak or no visual input will not cause provocation and the estimate depends mostly on vestibular and somatosensory inputs. The adaptive filterlike mechanism in the brain carries out a calibration of the two estimates to correct the erroneous weightings through graduated expansion of the foreground, to effect progressive habituation. This approach also allows the patients to use the stabilizing effect of the stationary background to resist the destabilizing effect from the foreground, so that a stronger stimulation in the foreground can be better tolerated and an effective training with more rapid escalation of intensity is possible. An additional advantage of this method is an objective measurement of the dizziness that can be used to evaluate the degree and change of a patient's dizziness while under treatment.
\end{abstract}

\section{Introduction}

Many patients suffer from dizziness that is triggered or aggravated by visual stimulation. This condition has been called visual vertigo (VV) [1,2], although usually the sensation of rotation (vertigo) is not present. Other terms describing this condition include space and motion discomfort [3], (inappropriate) visual dependence [4], and the "grocery store syndrome" [5]. While some patients have vestibular disorders or other organic conditions, many chronically dizzy patients have no abnormalities on detailed testing. It is believed that VV has been under-diagnosed leading to many dizzy patients not being properly treated [6].

Repeated optokinetic stimulation has been used for diagnosing and treating dizziness patients [7-10]. The authors [1] proposed a theoretical framework to treat VV patients that is also based on optokinetic stimulation but differs from both the traditional gaze stabilization and the virtual reality approach in vestibular rehabilitation. In this framework a stationary background is incorporated to the foreground stimulation. Here we will elaborate this theory and present the scheme as a continued calibration of sensory weights between the two regions and give graphical illustrations that can be easily understood and adapted in clinical settings.

\section{The Chang-Hain theory of progressive habituation}

The theory is based on the case of a patient whose VV started within days after the complete recovery from a benign paroxysmal positional vertigo (BPPV). During daytime and in a quiet environment without specific provocation or attacks the patient described his persistent dizziness at a very mild state as a feeling of floating. However, certain situations seemed to decrease this floating feeling so that the patient was briefly free from any dizziness symptoms. These situations all involved the presence of stationary and noncomplex objects. For example, he felt less floating outdoor around large and tall buildings or highway structures than in more open fields. Some especially interesting contrasts occurred in a car. As a driver he felt dizzier looking to the left than looking to the right, and as a passenger he felt dizzier in the front seat than in the back seat. There are findings [4] that VV is not related to a past history of motion sickness, but in this case the patient's experience directly counters the usual experience in motion sickness. Eventually he realized that both his driver's and passenger's experiences can be explained by the presence of stationary object in the field of view. At the driver seat that is located on the left side his view to the right was surrounded by a large part of the stationary frame inside the car. But when he looked to the left this stationary frame was a much smaller part of his view and he was much more exposed to the traffic outside the car. Likewise, as a passenger he saw less traffic and more of the inside frame of the car in the back seat than in the front seat.

${ }^{*}$ Correspondence to: Chih-Pei Chang, Department of Atmospheric Sciences, National Taiwan University, Taipei, Taiwan and Graduate School of Engineering and Applied Sciences, Naval Postgraduate School Code MR/Cp, Monterey, CA 93943-5114, USA, E-mail: cpchang@nps.edu

Key words: visual vertigo treatment, optokinetic, stationary anchoring

Received: August 07, 2020; Accepted: August 18, 2020; Published: August 25 , 2020 
The patient then devised an optokinetic stimulation exercise to experiment looking at moving trains briefly from the back seat of a car. He also experimented with train viewing at subway stations using the stationary platforms (Figure 1) to counteract the dizziness feelings. The exercises were conducted several times a day and he was able to gradually increase the duration from one or two seconds initially to longer periods. His symptoms started to subside after one week and completely disappeared after three weeks.

This case experience led us to hypothesize that the dizziness due to optical flow may be inversely correlated with the presence of stationary noncomplex images in the field of view. Since the degree of dizziness in this case depended on the difference between the destabilizing effect (D) of optical flow and the stabilizing effect (S) provided 5 by stationary objects in the visual field, we defined a destabilizing potential (DP):

$\mathrm{DP}=\mathrm{D} / \mathrm{S}$, which is the ratio of the destabilizing effect over the stabilizing effect. Figure 2 shows this scheme. Here a stationary point of regard, called aiming area $\mathrm{A}$, is embedded in a background environmental area $\mathrm{B}$, and $\mathrm{D}=\mathrm{A} \times \mathrm{f}(\mathrm{V})$ and $\mathrm{S}=\mathrm{B}$ where $\mathrm{V}$ is the velocity of the optical flow. In general $\mathrm{V}$ is a three-dimensional velocity field that may also vary with time, but for the simplest case of linear constant velocity,

\section{$\mathrm{DP}=\mathrm{D} / \mathrm{S}=\mathrm{A} / \mathrm{B} \times \mathrm{V}$}

The total effect on a VV patient is DP integrated over the duration of exposure,

\section{$\mathrm{TDP}=\int \mathrm{D} / \mathrm{S} \mathrm{dt}=\mathrm{t} \times \mathrm{A} / \mathrm{B} \times \mathrm{V}$ for constant $\mathrm{A}, \mathrm{B}$ and $\mathrm{V}$.}

The therapy (hereafter referred to as Chang-Hain or $\mathrm{CH}$ ) is to gradually increase the patient's tolerance of a higher TDP through exercises as a way to conduct progressive habituation. This can be done by increasing $\mathrm{V}$ and/or expanding $\mathrm{A}$ in figure 3 as the therapy progresses. This scheme may be described as an inverse of the traditional gaze stabilization exercises for vestibular rehabilitation in which the aiming area $\mathrm{A}$ is a small single object with slow relative motion, while the background environment is typically busy with fast optical flow or complex patterns (Figure 4). Figure 4 illustrates a patient under gaze stabilization therapy; a corresponding figure for the $\mathrm{CH}$ treatment is illustrated in figure 5 .

Due to miniature and possibly subconscious eye movements, the background is unlikely to stay perfectly stationary. To generalize the theory, we 04 may consider the background to contain a constant

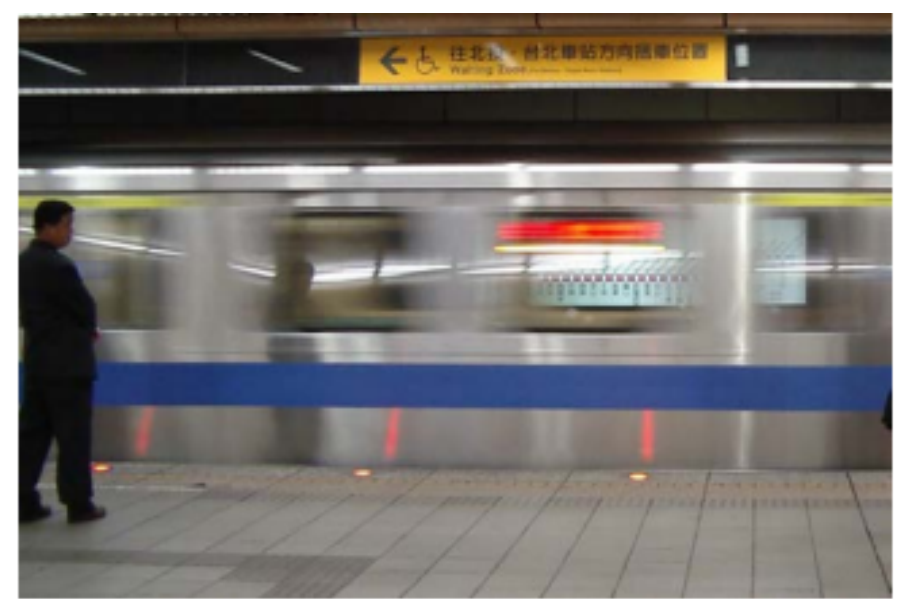

Figure 1. A moving train (fast optical flow that can provoke visual vertigo) passing through the stationary subway platform (stationary anchor in the field of view).

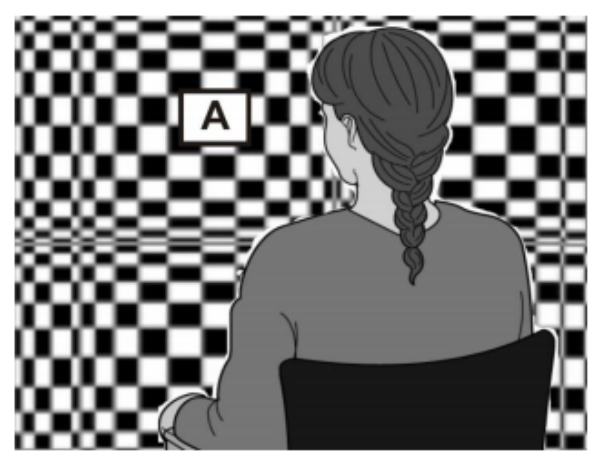

Figure 2. Illustration of traditional gaze stabilization therapy. The patient is aiming at a small noncomplex object $\mathrm{A}$ in a complex background that give rise to the optical flow.

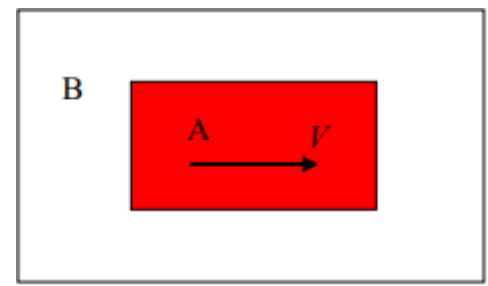

Figure 3. The Chang-Hain framework: Colored foreground contains optical flow (velocity V).

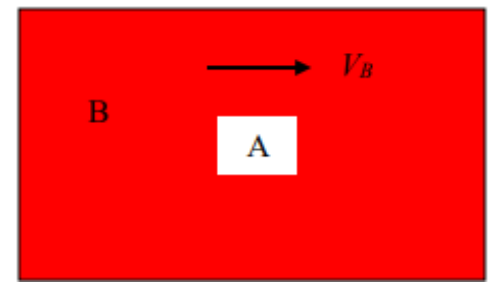

Figure 4. Gaze stabilization in vestibular rehabilitation therapy. Colored background contains optical flow (velocity VB).

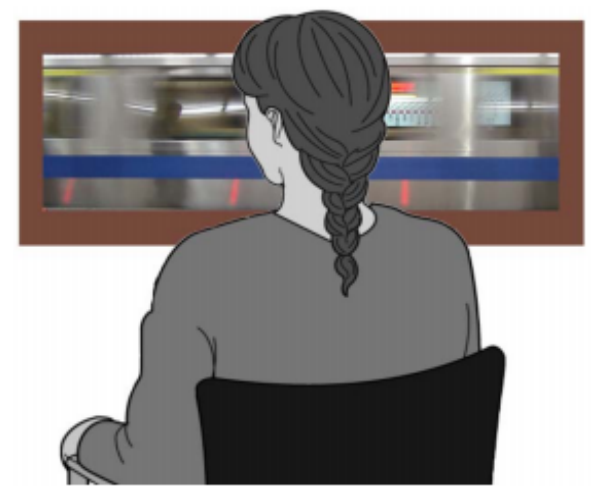

Figure 5. Illustration of the optokinetic stimulation with stationary anchoring at the frame. The patient uses the stationary frame to resist the provocation from the fast velocity in the aiming window.

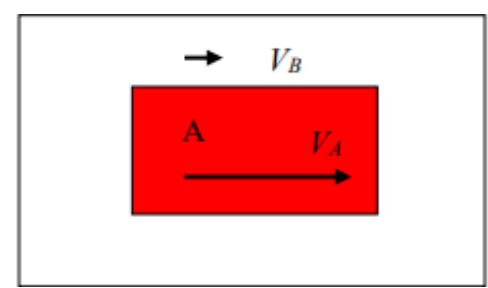

Figure 6. Similar to Figure 2 with strong optical flow VA in colored foreground and weak, non-provocative velocity VB in background. 
velocity $\mathrm{V}_{B}$ that is much smaller than $\mathrm{V}_{\mathrm{A}}$, as shown in $[4,6,20]$. In general, we may characterize $V_{B}$ as a velocity that tends to not provoke $\mathrm{VV}$ while $\mathrm{V}_{\mathrm{A}}$ as a velocity that tends to provoke VV. Again, consider a constant $\mathrm{V}_{\mathrm{A}}$, the velocity $\mathrm{V}$ in figure 2 is simply the relative velocity $\mathrm{V}=\mathrm{V}_{\mathrm{A}}-\mathrm{V}_{\mathrm{B}}$. A stationary anchor means a very small but probably nonzero $\mathrm{V}_{\mathrm{B}}$.

\section{Comparison to virtual reality and adaptive filtering}

Both the $\mathrm{CH}$ framework and the virtual reality (VR) training provide repeated optokinetic stimulation. The VR training including head mounted display (HMD), augmented, Fish Tank, and projectionbased [11] are all based on a computer-generated scenario with which the user can interact in three dimensions so that the user feels that he or she is part of the scene. In the HMD system the patient sees only the computer-generated image and is therefore totally immersed; in the projection-based system the field of view is wide to provide a similar sense of presence. In the other systems the field of view is limited but is compensated by the benefit of smaller pixel visual angles. In general, the promise of VR training is that the sense of presence in the training environment is an important factor for transferring into reality [11], thus total immersion in a controlled environment is desirable. The $\mathrm{CH}$ framework, on the other hand, is based on a limited field of view in the foreground that is accompanied by a stationary background. The latter is a critical element and the key difference from the traditional VR approach. Total immersion is purposely excluded.

The therapeutic effect of repeated optokinetic stimulation has been interpreted as due to the habituation by the brain [12]. The brain needs to combine visual, vestibular, and somatosensory inputs properly to provide a reasonable estimate of orientation in space and self and world motion. Many studies $[13,14]$ have reasoned that the mechanism of this sensorimotor processing is similar to that of an adaptive filter such as the Kalman filter in signal processing. A Kalman filter is a formal method of estimation of the true state of a system using an internal model. Internal models are used by the brain to construct percepts of the world especially in situations where sensory input may be redundant, intermittent, or unreliable. Disturbance of internal models have been postulated to account for one variant of motion sickness - mal de debarquement, that is commonly associated with visual dependence [15]. The architectural similarities between Kalman filter and the entorhinal-hippocampal loop and the neocortical hierarchy have been described $[16,17]$.

The adaptive internal model uses the difference between initial estimates based on a prediction model and actual sensory inputs to correct the estimate through an optimizing algorithm. In Kalman filter applications such as numerical weather prediction models, which use imperfect and incomplete observation meteorological data as initial conditions, the estimates and the inputs are assumed to have a normal probability distribution so that they can be represented by their means and standard deviations. The standard deviation is a measure of the noise level therefore the uncertainty of each quantity. In this process inputs from different sources are weighted according to a function of this uncertainty which depends on the uncertainty of each particular source relative to those of other sources. Quantities with large standard deviations are less reliable and receive a smaller weight, and the same process of weighting is applied in comparing and merging the initial prediction and actual inputs. For human sensorimotor processing, this process uses the prediction from the previous body state and corrects it with the current sensor inputs to arrive at the current body state.
For VV patients the brain inappropriately overweighs the visual inputs over the other, particularly vestibular inputs. This is often due to vestibular disease or neurological abnormality but can also develop in seemingly normal patients whose vestibular system is intact and free from any lesion in the peripheral or central nerve system. The patient in our previous study is one example [1]. It is likely that these patients who have experienced a vestibular insult in the past and the adaptive filter mechanism in the brain lowered the weighting of the vestibular input because it was very unreliable. After a patient recovers from the vestibular attack the brain is unable to adjust the weighs and continues to rely on visual inputs causing the inappropriate visual dependence and dizziness.

The VV patient that has recovered from a previous vestibular insult suffers from the wrong weighting. However, the brain is capable of real-time weight adjustment as demonstrated in a study of visual and auditory signals for spatial localization [19]. In the $\mathrm{CH}$ treatment theory, a VV patient with the wrong sensory weighting starts by getting provoked in a limited field of view in the foreground. Initially this field is small enough for the patient to tolerate the stimulation. This is analogous to conducting "nowcasting" in weather forecast in a small region that requires a computation power small enough to realize instantaneously. The stationary background provides several possible functions in this training. First, it provides a comfortable area for the patient to anchor the sight when the destabilizing effect of the stimulation is too stressful. Since the patient will not feel dizziness looking at this area, it serves as a kind of truth for the brain to calibrate the filter when the patient is viewing the two areas, one with provocation and one without, simultaneously. This is analogous to one aspect of the data quality control procedure used in weather forecast. Observational data at each point in the atmosphere are constantly compared with those in nearby points to assess their reliabilities. A sufficiently large background anchor area allows this calibration and reweighing to proceed with less resistance, reduces the chance that the patient's dizziness and intolerance interrupt the training process. After the patient's dizziness subsides, the total destabilizing potential is increased slightly by either increasing the foreground velocity or the foreground area or both. The patient's brain is now equipped with a somewhat improved weighting system with smaller errors in the sensory weights, and is therefore more likely to succeed in the next stage of training, in which the stabilizing effect is reduced while the destabilizing effect is increased. The key concept of the $\mathrm{CH}$ scheme is that this gradual increase of the destabilizing potential allows a phased calibration and adjustment of the sensory weightings. This approach may be easier than a full or wide field of view VR regimen to recover a state that was inappropriately locked in with overly strong visual dependence.

The $\mathrm{CH}$ process can also be compared to the virtual reality training of acrophobia through a gradual increase of the height [20]. Many acrophobia patients have an extreme fear of height because of excessive visual dependence. They experience difficulty as the increased distance from ground causes them to lose reference in space, which is equivalent to a large $\mathrm{A} / \mathrm{B}$ ratio for the VV patients. The gradual increase of height in the visual reality training of the acrophobia patients may therefore be interpreted as an equivalence of the gradual increase of the destabilizing potential.

\section{Conclusion}

The effect of incorporating a stationary anchor background in the optokinetic stimulation therapy of VV patients, who suffer from 
dizziness provoked by optical flow, offer several potential benefits. Correction of the errors in the overweighing of visual inputs and under-weighing of vestibular and somatosensory inputs may be easier when the adaptive filter-like mechanism in the brain compares the estimates from the foreground to that of the background. In the foreground a higher velocity provides strong optical simulation and may provoke a dizziness attack, while the background by itself will not cause provocation because the visual input is weak or near absent and the estimate depends mostly on vestibular and somatosensory inputs. This calibration of the two estimates may help the adaptive filtering process to correct the erroneous weightings that may be the result of past vestibular insults. Moreover, the $\mathrm{CH}$ framework allows the patients to use the stabilizing effect of the stationary background to resist the destabilizing effect from the foreground, so that the patient can better tolerate a stronger stimulation in the foreground.

The method can also be adopted economically by training the patients to practice in everyday situations, such as watching a moving train in a properly anchored background environment (Figure 1). The basic framework of calculating a destabilizing potential with the ratio of the foreground and background areas and the difference between their relative velocities also provide a simple, objective way to calculate the visual dependence threshold of a VV patient, which is valuable since available objective and consistent diagnostic tools for measuring the degree of dizziness are very limited [21].

\section{Acknowledgements}

We wish to thank Ms. Mei-Yun Wang for preparing the figures. This work was supported in part by MOST 109-2111-M-002-004project at National Taiwan University.

\section{Author Disclosure Statement}

No competing financial interests exist.

\section{References}

1. Chang CP, Hain TC (2008) A theory for treating dizziness due to optical flow. Cyberpsychol Behav 11: 495-498. [Crossref]

2. Bronstein AM (1995) The visual vertigo syndrome. Acta Otolaryngol Suppl 1: 45-48. [Crossref]

3. Jacob RG, Lilienfeld SO, Furman JM, Durrant JD, Turner SM (1989) Panic disorder with vestibular dysfunction: Further clinical observations and description of space and motion phobic stimuli. J Anx Dis 3: 117-130.

4. Guerraz M, Yardley L, Bertholon P, Pollak L, Rudge P, et al. (2001) Virtual Reality symptom assessment, spatial orientation and postural control. Brain 124: 1646-1656. [Crossref]
5. Hester RB 3rd, Farris BK (1991) Acetazolamide in the treatment of abnormal oculovestibular response. Am J Ophthalmol 111: 215-220. [Crossref]

6. Bronstein AM (2002) Under-rated neuro-otological symptoms: Hoffman and Brookler 1978 revisited. Br Med Bull 63: 213-221. [Crossref]

7. Virre E (1996) Virtual reality and the vestibular apparatus. IEEE Engi Medi Biol Magazine 15: 41-69.

8. Sparto PJ, Whitney SL, Hodges LF, Furman JM, Redfern MS (2004) Simulator sickness when performing gaze shifts within a wide field of view optic flow environment: preliminary evidence for using virtual reality in vestibular rehabilitation. J Neuroengineering Rehabil 1: 14. [Crossref]

9. Whitney SL, Sparto PJ, Hodges LF, Babu SV, Furman JM, et al. (2006) Responses to a virtual reality grocery store in persons with and without vestibular dysfunction. Cyberpsychol Behav 9: 152-156. [Crossref]

10. Keshner EA, Streepey J, Dhaher Y, Hain T (2007) Pairing virtual reality with dynamic posturography serves to differentiate between patients experiencing visual vertigo. $J$ Neuroeng Rehabil 4: 24. [Crossref]

11. Keshner EA (2004) Virtual reality and physical rehabilitation: a new toy or a new research and rehabilitation tool? J Neuroeng Rehabil 1: 8. [Crossref]

12. Toh EH (2008) Vertigo and Disequilibrium A Practical Guide to Diagnosis and Management. Thieme.

13. Rao R, Ballard D (1999) Predictive coding in the visual cortex: A functional interpretation of some extra-classical receptive-field effects. Nat Neurosci 2: 79-87. [Crossref]

14. Goussev V (2004) Does the brain implement the Kalman filter? Behav Brain Sci 3: 404-405.

15. Hain TC, Helminski JO (2007) Mal de Debarquement. Vestibular Rehabilitation. Philadelphia: FA. Davis Company.

16. Lörincz A, Buzsááki G (2000) The parahippocampal region: Implications for neurological and psychiatric diseases. In Scharfman H, Witter M, Schwarz R (eds). Annals of the New York Academy of Sciences. New York: New York Academy of Sciences pp. 83-111.

17. Lörincz A, Szatmáry B, Szirtes G (2002) Mystery of structure and function of sensory processing areas of the neocortex: A resolution. J Comp Neurosci 13: 187-205. [Crossref]

18. Zhou X (2004) Some concepts and methods of atmospheric data assimilation. Observation, Theory and Modelling of Atmospheric Variability. Singapore: World Scientific, pp. 234-276.

19. Battaglia PW, Jacobs RA, Aslin RN (2003) Bayesian integration of visual and auditory signals for spatial localization. J Opt Soc Am A Opt Image Sci Vis 20: 1391-1397. [Crossref]

20. Emmelkamp PM, Bruynzeel M, Drost L, Van Der Mast C (2001) Virtual reality treatment in acrophobia: a comparison with exposure in vivo. CyberPsychol Behav 4: 335-339. [Crossref]

21. Pavlou M, Davies RA, Bronstein AM (2006) The assessment of increased sensitivity to visual stimuli in patients with chronic dizziness. J Vestib Res 16: 223-231. [Crossref]

Copyright: (C2020 C-P Chang. This is an open-access article distributed under the terms of the Creative Commons Attribution License, which permits unrestricted use, distribution, and reproduction in any medium, provided the original author and source are credited. 